\title{
A Static Study of Java Exceptions Using JESP ${ }^{\star}$
}

\author{
Barbara G. Ryder, Donald Smith, Ulrich Kremer, \\ Michael Gordon, and Nirav Shah \\ Department of Computer Science, Rutgers University \\ 110 Frelinghuysen Road, Piscataway, NJ 08854-8019, USA \\ fax: 732-445-0537 \\ \{ryder, dsmith, uli, fabfour, nshah\}@cs.rutgers.edu
}

\begin{abstract}
JESP is a tool for statically examining the usage of user thrown exceptions in Java source code. Reported here are the first findings over a dataset of 31 publicly available Java codes, including the JavaSpecs. Of greatest interest to compiler writers are the findings that most Java exceptions are thrown across method boundaries, trys and catches occur in equal numbers, finallys are rare, and programs fall into one of two categories, those dominated by throw statements and those dominated by catch statements.
\end{abstract}

\section{Introduction}

Java already has been embraced as a Web programming language and is beginning to gain acceptance as a language for general applications. In response to the acceptance and use of this and other OO languages, compile-time analysis and code transformations that produce optimized code for features found in such languages(e.g., polymorphism, exceptions, etc.) are being studied. This paper summarizes how exceptions are used in current Java codes. The information gathered shows that exceptions are ubiquitous and that their use often falls into specific patterns.

Exception handling is common in Java programs for the Web, but it is also true that exceptions will play a significant role in general purpose applications. The use of exceptions in general applications is due to several emerging trends. Key among these are: the development of automated systems with complex control paths, and the shift toward exception-based programming paradigms seen in most introductory language and data-structures texts.

Automated systems are being built around legacy codes that were designed to be controlled by humans and are now being controlled by programs. Not surprisingly, human-friendly codes are proving to be program-unfriendly, necessitating the adaptation of these codes for use under program, as opposed to human, control. These legacy codes have been built over several years by several people, have been validated against extensive test suites, and are now trusted tools. Revalidation of such codes is very expensive in time and money thus constraining adaptations of these codes to minimize the need for revalidation. One

\footnotetext{
* The research reported here was supported, in part, by NSF grant CCR-9808607.
} 
of the most promising strategies to facilitate the adaptation of legacy codes under the constraint of limited revalidation is the introduction of wrappers $[12,11,8]$ that handle unexpected situations. Wrappers provide a mechanism that detects when a code has failed and passes control to a module designed to manage the failure. Java exceptions, in conjunction with their catch and throw operators, provide an ideal mechanism for implementing wrappers.

In addition to the development wrapper-based automated systems, a general shift toward exception-based programming paradigms is being observed. There are several reasons for this shift including program correctness, program clarity, and elimination of side effects. These reasons have been noted by many in the community. Texts and references now contain persuasive arguments, such as the following.

Exceptions provide a clean way to check for errors without cluttering code. Exceptions also provide a mechanism to signal errors directly rather than use flags or side effects such as fields that must be checked. Exceptions make the error conditions that a method can signal an explicit part of the method's contract. $p .151[1]$

These two trends, along with the growth in popularity of object-oriented languages such as Java, make it clear that exceptions will play an increasing role in program design, and thus present a new challenge to optimizing compilers.

The Java exception model is very strict as to what program state must be made available to the user when an exception occurs.

Exceptions in Java are precise: when the transfer of control takes place, all effects of the statements executed and expressions evaluated before the point from which the exception is thrown must appear to have taken place. No expressions, statements, or parts thereof that occur after the point from which the exception is thrown may appear to have been evaluated. If optimized code has speculatively executed some of the expressions or statements which follow the point at which the exception occurs, such code must be prepared to hide this speculative execution from the user-visible state of the Java program. p.205[9]

In order to produce efficient optimized code that meet these specifications, safety conditions must be guaranteed for any optimizing transform used by the compiler. Since exceptions produce new and distinctive control-flow patterns, new static analysis techniques must be developed that accurately estimate control flow $[5,4,6,10,14]$. Speculation of instructions must be consistent with what is required by this exception model[2,13,7]. Practical compile-time analyses and code transformations should take advantage of common patterns of exception usage, especially in calculating possible profitability of transformations or the appropriateness of approximation in analysis.

We partition exceptions into two classes: those that are not usually thrown by a user and those that are usually thrown by a user. The former are usually thrown by the runtime system and are objects in the RuntimeException class; 
for example, NullPointerException and ArrayBoundsException are among these. The latter usually appear in a throw statement of a user's Java program and are often, but not always, a user-defined subclass of Exception. Both kinds of exceptions complicate program control flow. The empirical study reported here is an examination of Java program source codes using the Prolangs JESP tool to determine their patterns of user thrown exceptions. These initial results (and further planned static and dynamic studies) will serve as a basis for focusing compiler writers' attention on those uses of exceptions which actually occur in real codes. Additionally, it will allow compiler writers to de-emphasize those that may be difficult to transform, but do not occur very often (e.g., nested finallys).

Outline. Section 2 presents the dataset used in this study. The JESP tool and empirical measurements obtained in the study are explained and interpreted in Section 3. Ramifications of the findings for optimizing compilers are listed in Section 4. Related work is mentioned in Section 5. Section 6 states the conclusions and briefly describes future work.

\section{Description of Data}

As an initial study, a representative set of popular Java applications and applets were gathered. Included are a large number of programs from JARS, the Java Review Service, ${ }^{1}$ rated in their top twenty percent by utility. These programs include:

- IceMail - a Java email client

- JFlex - a lexical analyzer generator

- RabbIT - a web proxy

- WebHunter - a file downloader

- LPR- a class library that allows printing to a standard LPD (Unix) printer over the network.

- Creature - an artificial life simulator

- Statistician - lists method statistics for a class

- Vigenere - encrypts text based on the vigenere algorithm

- JavaBinHex - a BinHex decompresser

- JHLZip - compresses an uncompressed zip file

- JHLUnzip - decompresses a compressed zip file

- Othello - a Reversi applet

A large number of Java programs that are currently being developed also were included. Among these programs are McGill University's Soot beta 4[15], a Java bytecode analysis and transformation framework and its accompanying tools. Additional programs, such as the Hot Path Browser ${ }^{2}$ and JavaCC Bame $^{3}$ cam

1 http://www.jars.com

2 http://www.bell-labs.com/project/HPB/

3 http://www.suntest.com/JavaCC/ 
from Lucent Technologies/Bell Laboratories and Sun Microsystems, respectively. The Hot Path Browser allows the user to view the most frequently executed paths of a program given the correct path information. JavaCC is a parser generator. The final two programs are from SBKTech; Jas a Java bytecode assembler and Jell a parser generator.

\section{$3 \quad$ Findings}

\subsection{Experimental Framework}

To gather the static measurements of the sample set of applications, a set of tools collectively known as JESP (Java Exception Static Profiler) were built using the JTrek library from Compaq/Digital ${ }^{4}$ The $\mathbf{J T r e k}$ library is a Java API that allows developers to instrument, profile, and decompile Java classes. It allows for rapid development of both static and dynamic profiling tools. Specifically, the Trek class library models Java code as a hierarchy of objects. The Trek API allows a developer to scan through a program's bytecode instructions, with classes representing the program's class files, and the fields, methods, local variables, statements, and instructions within them.

\subsection{Data Gathered}

The 31 Java programs evaluated in this study are shown in Figure 1. They were obtained from JavaSpecs ${ }^{5}$ as well as from public sources on the Internet and ranged in size from 48 to 96,000 lines of code. JESP analyzed each program and produced summaries of exception usage and structure based on JDK 1.1.5. The usage and structure of exceptions and exception constructs are reported in the sections that follow.

General Descriptive Data Figure 1 describes the programs that were evaluated. The codes are ordered from smallest to largest based on number of lines of code. Additional fields giving size in bytes, number of classes ${ }^{6}$, number of classes with an implements clause, total number of methods, and percentage of methods with exception constructs (i.e., throw, try, catch, finally) are also shown.

Examination of Figure 1 shows that multiple inheritance, as indicated by an implements clause, is used infrequently in smaller programs of the dataset (i.e., under 5,000 lines of code). Figure 2(a) shows that lines of code per class has little or no correlation with program size and Figure 2(b) corroborates the prevailing view that Java methods are short. For the programs examined the number of lines of code per method had little or no correlation with program size and averaged 33 (median 26) over all programs. Note: Statistician (id 7, with 6996 lines of code) is an outlier because it has only one class.

\footnotetext{
${ }^{4}$ http://www.digital.com/java/download/jtrek/index.html

${ }^{5}$ http://www.specbench.org

${ }^{6}$ Figure 1 list number of files. In Java, files and non-internal classes are 1-1 mappable.
} 


\begin{tabular}{|c|c|c|c|c|c|c|c|}
\hline & Program & $\begin{array}{r}\text { Lines of } \\
\text { Code }\end{array}$ & Bytes & $\begin{array}{r}\text { Files } \\
\text { Analyzed }\end{array}$ & Implements & $\begin{array}{r}\text { Total } \\
\text { Methods }\end{array}$ & $\begin{array}{r}\% \text { of Meth. } \\
\text { w/ Exceptions }\end{array}$ \\
\hline 1 & $227 \mathrm{mtrt}$ & 48 & 859 & 1 & 1 & 4 & $0 \%$ \\
\hline 2 & JHLUnzip & 187 & 3,262 & 1 & 0 & 3 & $67 \%$ \\
\hline 3 & spec & 283 & 5,420 & 2 & 0 & 11 & $18 \%$ \\
\hline 4 & \begin{tabular}{|l|} 
JavaBinHex \\
\end{tabular} & 300 & 4,851 & 1 & 0 & 4 & $50 \%$ \\
\hline 5 & \begin{tabular}{|l|} 
Vigenere \\
\end{tabular} & 305 & 9,333 & 3 & 2 & 22 & $18 \%$ \\
\hline 6 & 999 checkit & 332 & 5,899 & 3 & 1 & 8 & $25 \%$ \\
\hline 7 & Statistician & 387 & 6,996 & 1 & 0 & 2 & $50 \%$ \\
\hline 8 & \begin{tabular}{|l|}
$J H L Z i p$ \\
\end{tabular} & 425 & 5,473 & 2 & 0 & 11 & $27 \%$ \\
\hline 9 & Javacc-JJTree-JJDoc & 602 & 21,573 & 6 & 0 & 22 & $14 \%$ \\
\hline 10 & LPR & 777 & 14,731 & 3 & 0 & 52 & $25 \%$ \\
\hline 11 & 201 compress & 927 & 17,821 & 12 & 1 & 44 & $2 \%$ \\
\hline 12 & $209 \mathrm{db}$ & 1028 & 10,156 & 3 & 1 & 34 & $21 \%$ \\
\hline 13 & WebHunter & 1610 & 36,114 & 6 & 9 & 81 & $11 \%$ \\
\hline 14 & 200_check & 1818 & 40,381 & 17 & 7 & 107 & $19 \%$ \\
\hline 15 & io & 2631 & 58,057 & 8 & 0 & 98 & $13 \%$ \\
\hline 16 & reporter & 2743 & 71,703 & 18 & 3 & 114 & $21 \%$ \\
\hline 17 & Othello & 2804 & 41,427 & 18 & 0 & 164 & $9 \%$ \\
\hline 18 & 205_raytrace & 3751 & 57,000 & 25 & 1 & 176 & $4 \%$ \\
\hline 19 & harness & 4882 & 83,899 & 23 & 2 & 185 & $12 \%$ \\
\hline 20 & \begin{tabular}{|l|} 
Jas \\
\end{tabular} & 4920 & 210,807 & 121 & 89 & 422 & $14 \%$ \\
\hline 21 & RabblT & 5276 & 128,552 & 43 & 15 & 267 & $17 \%$ \\
\hline 22 & \begin{tabular}{|l|} 
Joie \\
\end{tabular} & 6773 & 147,757 & 63 & 3 & 630 & $8 \%$ \\
\hline 23 & Soot-SableUtil & 7155 & 86,006 & 67 & 23 & 484 & $12 \%$ \\
\hline 24 & \begin{tabular}{|l|}
202 jess \\
\end{tabular} & 10579 & 396,536 & 151 & 106 & 690 & $11 \%$ \\
\hline 25 & \begin{tabular}{|l|l|} 
Jell \\
\end{tabular} & 10747 & 140,252 & 51 & 20 & 337 & $1 \%$ \\
\hline 26 & Creature & 11142 & 98,932 & 46 & 15 & 152 & $4 \%$ \\
\hline 27 & HotPath Browser & 11512 & 259,697 & 108 & 43 & 891 & $5 \%$ \\
\hline 28 & Jflex & 11796 & 192,839 & 53 & 16 & 420 & $7 \%$ \\
\hline 29 & Soot-Jasmin & 17152 & 253,292 & 99 & 22 & 664 & $8 \%$ \\
\hline 30 & \begin{tabular}{|l|} 
ICEMail \\
\end{tabular} & 25974 & 457,246 & 135 & 72 & 966 & $13 \%$ \\
\hline 31 & Soot beta 4 & 96144 & $1,033,630$ & 651 & 276 & 3096 & $4 \%$ \\
\hline
\end{tabular}

Fig. 1. Programs in dataset

Try, catch, throw, and finally statements This section reports on constructs that play a role in exception processing. JESP totals the number of try, catch, throw and finally statements along with the number of throws declarations used in a program. Methods declared to throw multiple exceptions are handled the same as methods declared to throw only one exception - each are counted only once.

Across the entire dataset, Figure 3 shows that on average 16\% (median 13\%) of the methods contain some kind of exception construct and that this percentage is insensitive to program size. This confirms the belief that exceptions will be ubiquitous in Java programs and highlights the fact that optimization transformations which are effective vis-a-vis exceptions will be necessary for efficient code generation.

Figures 4(a) and 4(b) display how many catch, throw and try statements occur in the dataset. Results for finally statements are not presented in the figure since they are rarely used; 26 of the 31 programs do not contain any finally clause. Similarly, throws were rare in the smaller programs; none of the programs smaller than 1615 lines of code contained a throw. Note that the number of catch and try statements in each program seem equal. 


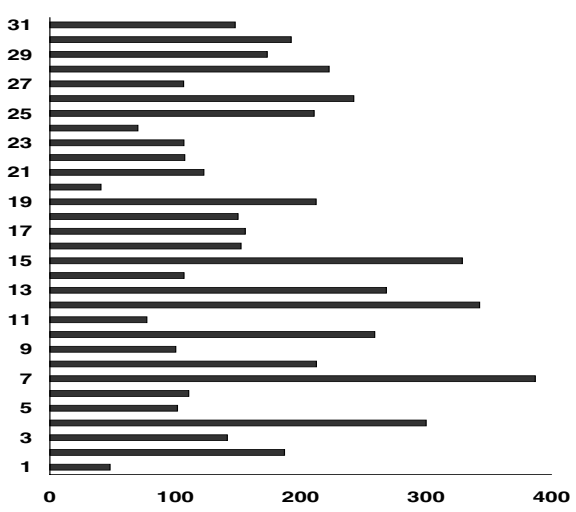

(a)Lines per Class

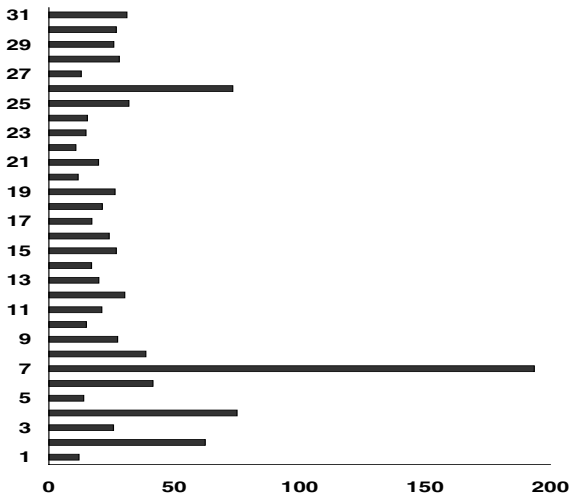

(b) Lines per Method

Fig. 2. Average number of lines of code

Figure 5 displays the ratio of try to catch statements. $50 \%$ of the programs have exactly 1 catch per try and only 1 program exceeds 1.5 catches per try. This implies that try clauses almost always contain 1 catch statement and very rarely contain 2 or more catch statements. The fact that trys usually contain only 1 catch provides strong evidence that specialization and inlining transformations may be effectively employed to improve performance.

A surprising feature of our dataset is that almost every program is dominated by either catches or throws and seldom have similar numbers of each. Figure 6 shows the breakdown by percent of catch and throw statements. Only one procedure, io (id 16, with 2632 lines of code), has the number of throws and catches nearly equal. In all other cases the ratio of catches to throws is greater than 3-to-1 or less than 1-to-3. This is indicative of a difference in the kinds of applications being measured. Applications with more throws than catches are hypothesized to be libraries, which must be robust over all possible uses of their methods. Similarly, applications with more catches than throws are probably clients of libraries that are handling the exceptions returned by library routines rather than passing them up the Java runtime stack. This, along with lack of finally statements, indicates that exceptions are fully processed where, and when, they are first caught.

We were very interested in seeing how exceptions are used in finally clauses and to see if potential complications to control flow were observed in practice. Unfortunately finally statements were so rare in our dataset that reporting on exceptions in finally statements is impossible.

Distance between a throw and its corresponding catch JESP was used to measured specific patterns of exception constructs within try statements and to report the number of throws not enclosed in a try statement. We considered four distinct usage patterns for try statements. Trys that contain: 


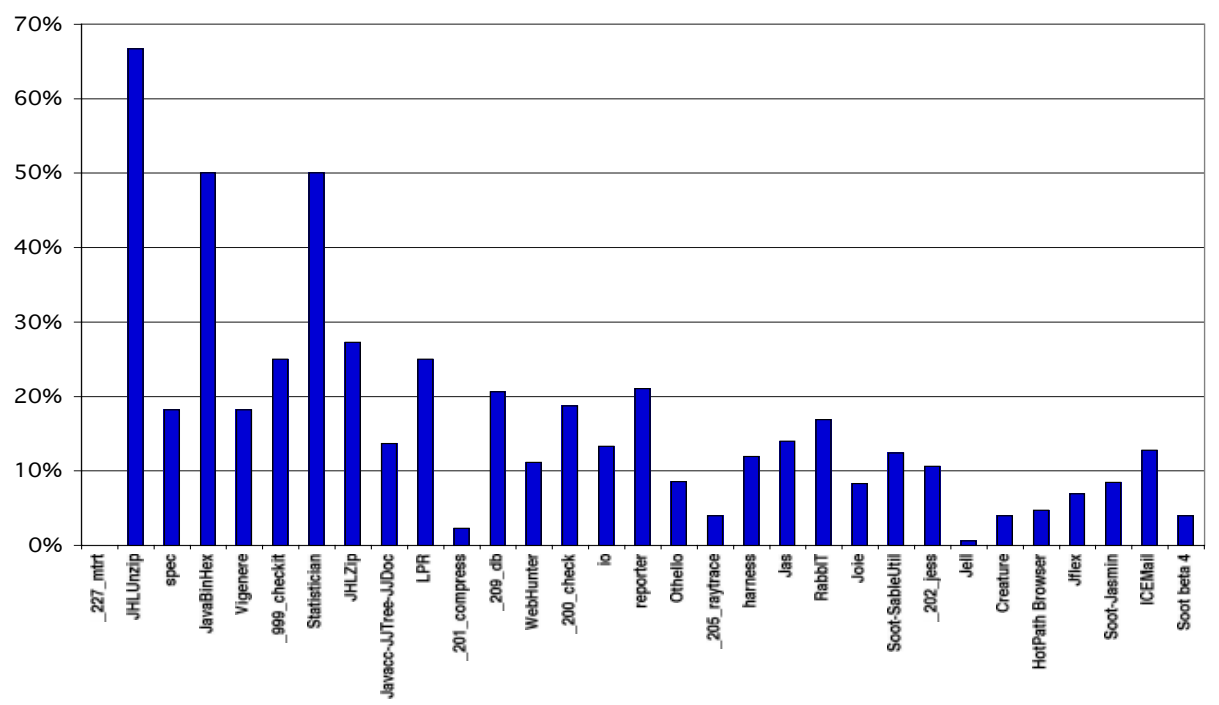

Fig. 3. Percentage of methods with try, catch, and throw constructs

- one or more throws and a corresponding catch,

- no throws and a catch,

- one or more throws and no catches, and

- no throws and no catches

Each try construct could optionally contain a finally; however for the dataset evaluated this had little impact. We found, as expected, that throws do not occur in try statements but rather occur outside of try statements. Figures 7(a) and 7(b) summarize our data. These observations support our belief that exception handling seems to be done by passing the exception up the current call stack. A try without a throw probably contains a method call that may throw an exception. ${ }^{7} \mathrm{~A}$ throw not in a try is probably in a method called directly or indirectly from a try block. Thus, the next question to be investigated both statically and dynamically is "How far up the call stack is an exception object passed, on average?" This knowledge will aid in tailoring optimizations of this exception usage, especially for non-aborting control-flow exceptions.

Characterizing Exceptions JESP was also used to categorize the class of the object that is being thrown as either a Java-defined exception, User-defined exception, or Unknown. A Java-defined exception is an instance of an exception class that is defined in the JDK 1.1.5. User-defined exceptions are classified as

\footnotetext{
${ }^{7}$ It is possible for a try block to be used to catch exceptions such as ArrayBoundsException or other subclasses of RuntimeException which can be generated without a method call; however, we believe this use of try blocks is very rare.
} 


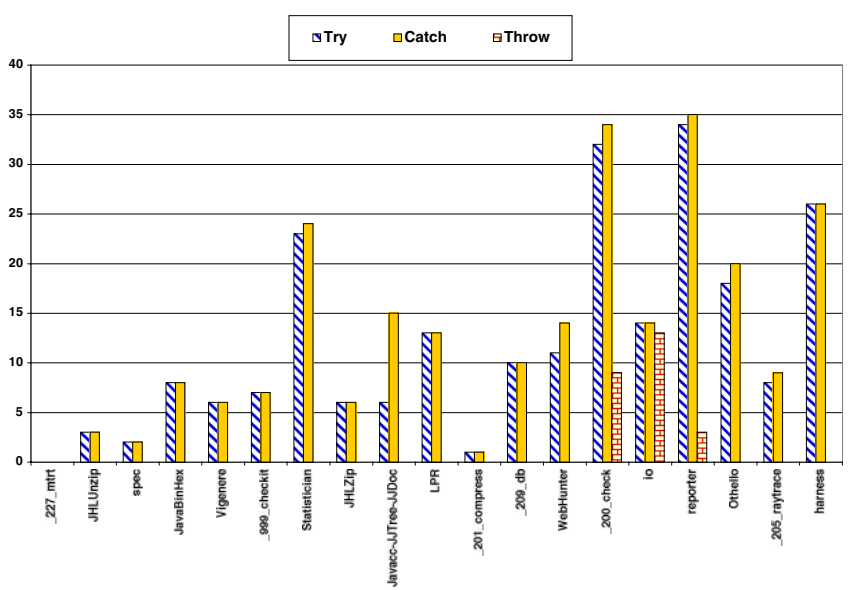

(a)Small Programs(under 5000 lines)

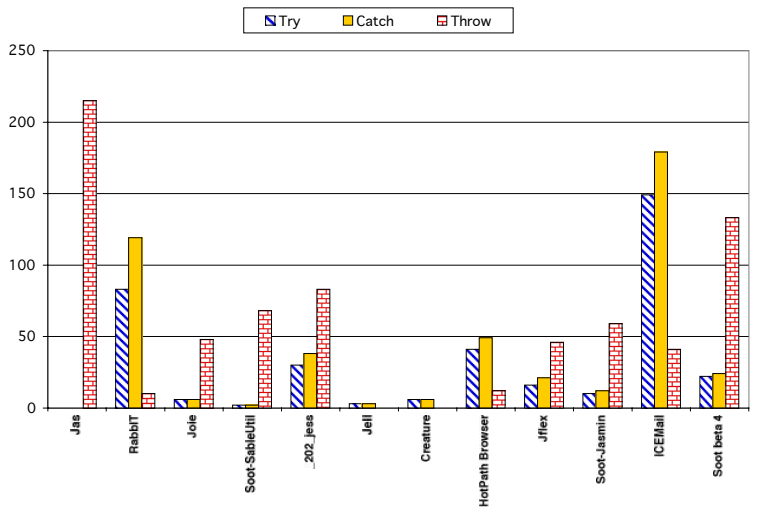

(b)Large Programs(over 5000 lines)

Fig. 4. Try, catch, throw counts per program

exception objects that are instances of classes not defined in the JDK. These can include exception classes defined in any library code that the program uses. Figure 8 reports these results and clearly shows that user defined exceptions are much more prevalent than Java-defined exceptions. In two cases, library code was not available and we found programs that did not define an exception class yet did throw one or more user-defined exceptions. These are not included in Figure 8.

The prevalence of user-defined exceptions is strong supporting evidence that exceptions are not only ubiquitous but also gaining acceptance as a mainstream control flow mechanisms in Java. The implications are clear for optimizing compilers. They will have to produce efficient code from source that contains userdefined exceptions and catch/throw patterns that manage control flow. 


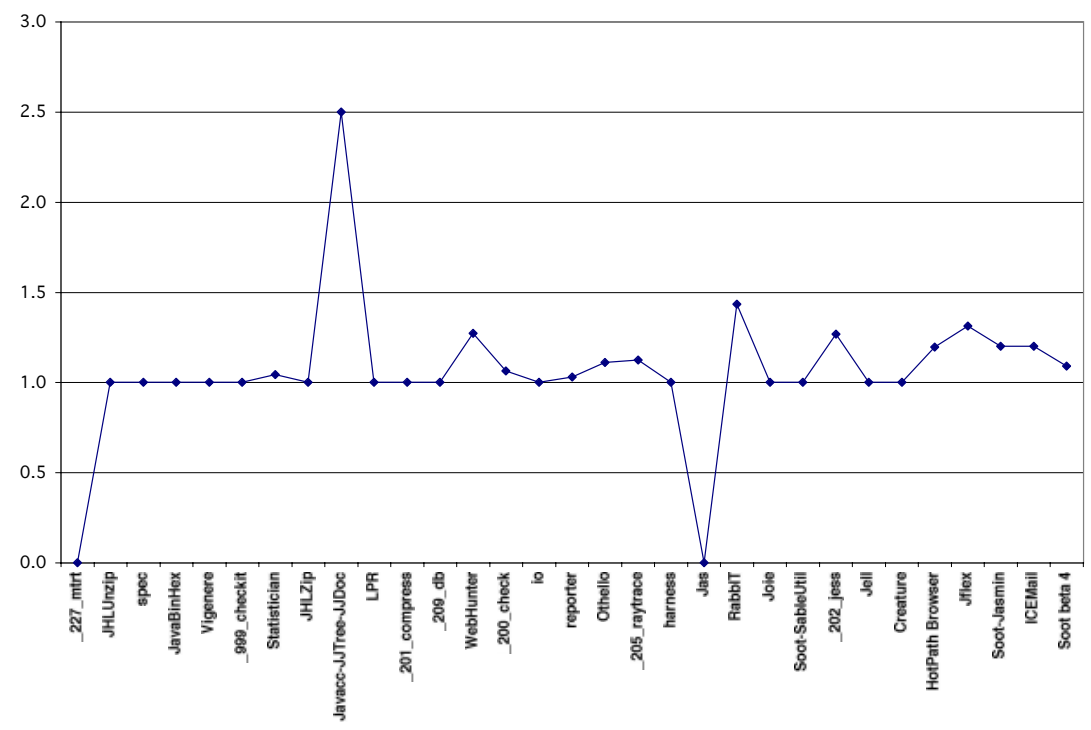

Fig. 5. Average number of catches per try

The final category of data that JESP gathered is information regarding the number of exception classes that the program contains as well as the shape of the exception tree hierarchy that the program defines. An exception class was considered to be any class that extends Throwable or any of its subclasses, excluding classes that extend Error. To analyze the shape of the exception tree, JESP gathers the maximum breadth and depth of the tree. Results of this analysis indicate that user-defined exceptions change the exception tree very little. Without any user-defined exceptions, the JDK's base exception tree has a maximum breadth of eleven and a maximum depth of four. With user-defined exceptions the breadth and depth changed very little if at all. More work is needed to separate the user- and Java-defined trees so representative figures can be reported. Preliminary indications are that user-defined exception hierarchies are very shallow (i.e., in our dataset no program had a user-defined hierarchy deeper than 2).

\section{Ramifications}

The gathered statistics about the use of exceptions in Java are preliminary. More experiments, in particular dynamic program instrumentation, are needed to give a more detailed picture of exceptions and their use in Java. However, some interesting conclusions and conjectures can already be made.

- finally clauses are only used rarely in our benchmark suite. This suggests that the development of techniques to optimize finally clauses and handle 


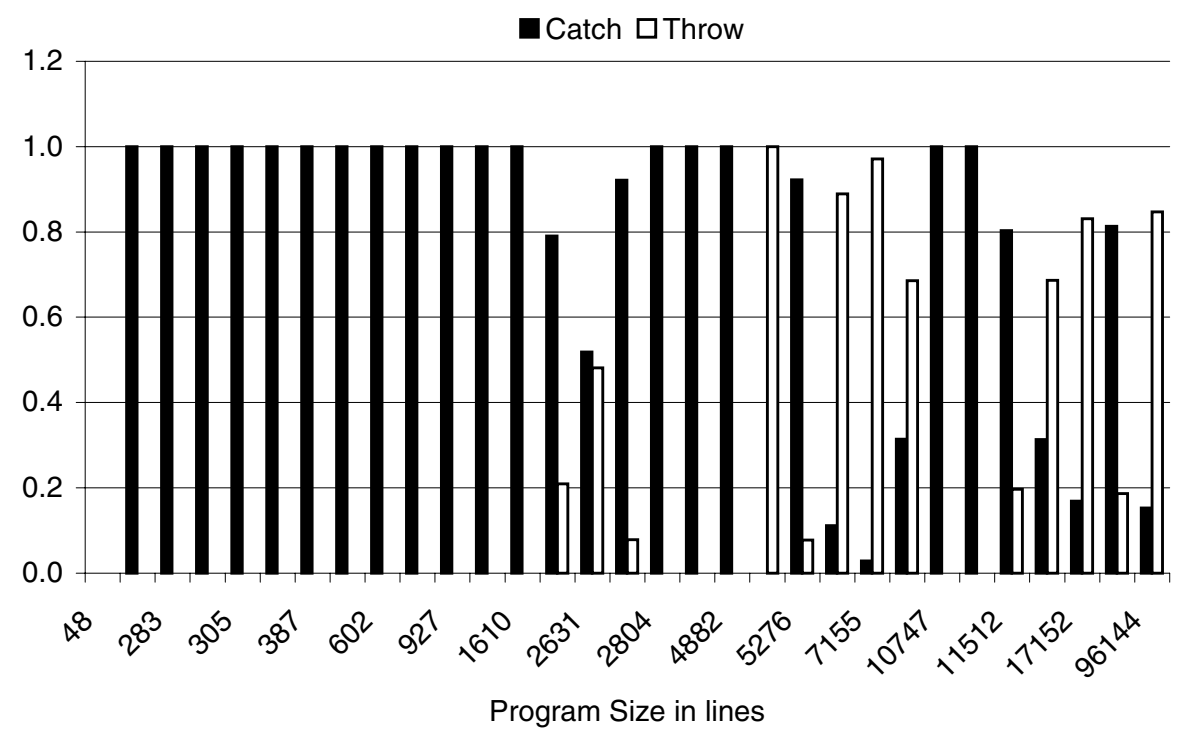

Fig. 6. Catch vs. Throw

the potential complexity they introduce into exception handling may not be profitable.

- The typical try blocks contains only one catch clause. This suggests that users have a particular exception event in mind when writing a typical try block and indicates a local treatment of exceptions may be possible. In such cases a compiler, supported by the appropriate analysis, may be able to determine which catch clause will process an exception thrown from a specific program point.

- The combination of throw statements being rare in try blocks, try blocks containing only one catch each, and the user-defined exception hierarchy being shallow indicates that the static and dynamic distance between throw and catch events in the call graph or on the run time stack may be short.

- There are a significant number of explicit throw statements outside of try blocks. This suggests that exception handling cannot be dealt with inside a single method. However, more work is needed to determine if exceptions are handled within methods of the same class or passed across classes. If exceptions are thrown and caught in the same class more aggressive analyses and optimizations are possible.

In Section 1 exceptions were classified as either usually thrown by a user or usually not thrown by a user; this study gathers information about the former. There is another useful classification of exceptions as either abortive or control exceptions. Abortive exceptions do not allow program recovery, but lead to the termination of the program, possibly after some clean-up or diagnostic analysis 


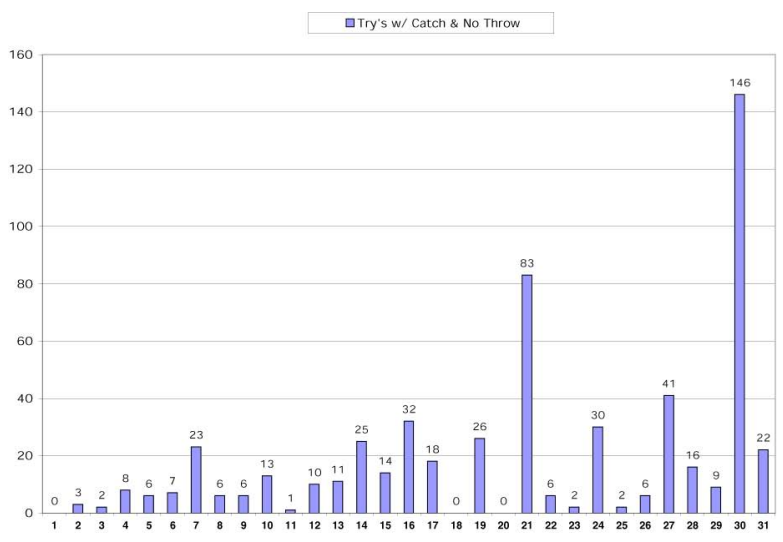

(a) Trys with catches but no throws

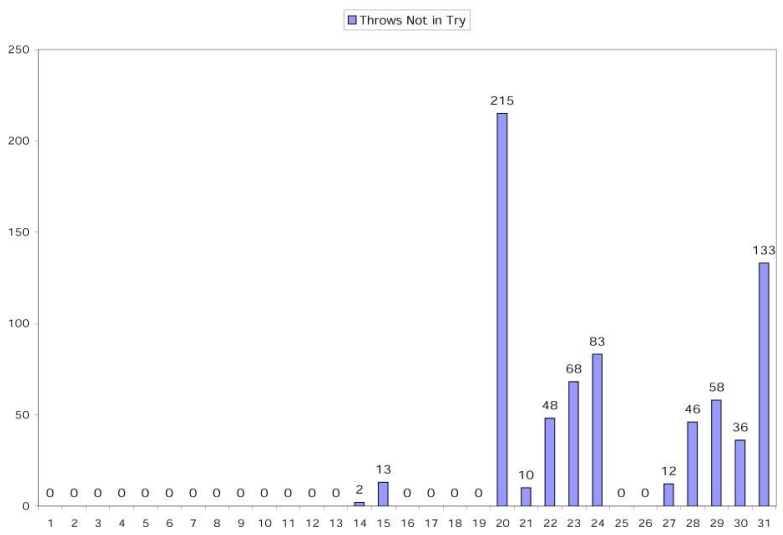

(b) Throws not in try block

Fig. 7. Exception constructs

has been performed in the corresponding catch clause. In contrast, a control exception signals some abnormal, but anticipated program condition from which the program is expected to recover. In this case, the corresponding catch clause contains recovery code and initiates the continuation of program execution in an updated program state.

Since abortive exceptions lead to program termination, the benefit of optimized code for these exceptions is minimal. Since they are abortive, their expected frequency should be rather low in debugged and tested codes and any optimization will only speedup the final cleanup stages before termination. Therefore, efforts to optimize the execution of an abortive exception itself are not beneficial. This is not true for control exceptions since they represent a likely control flow path in the program. An aggressive optimizing compiler should op- 


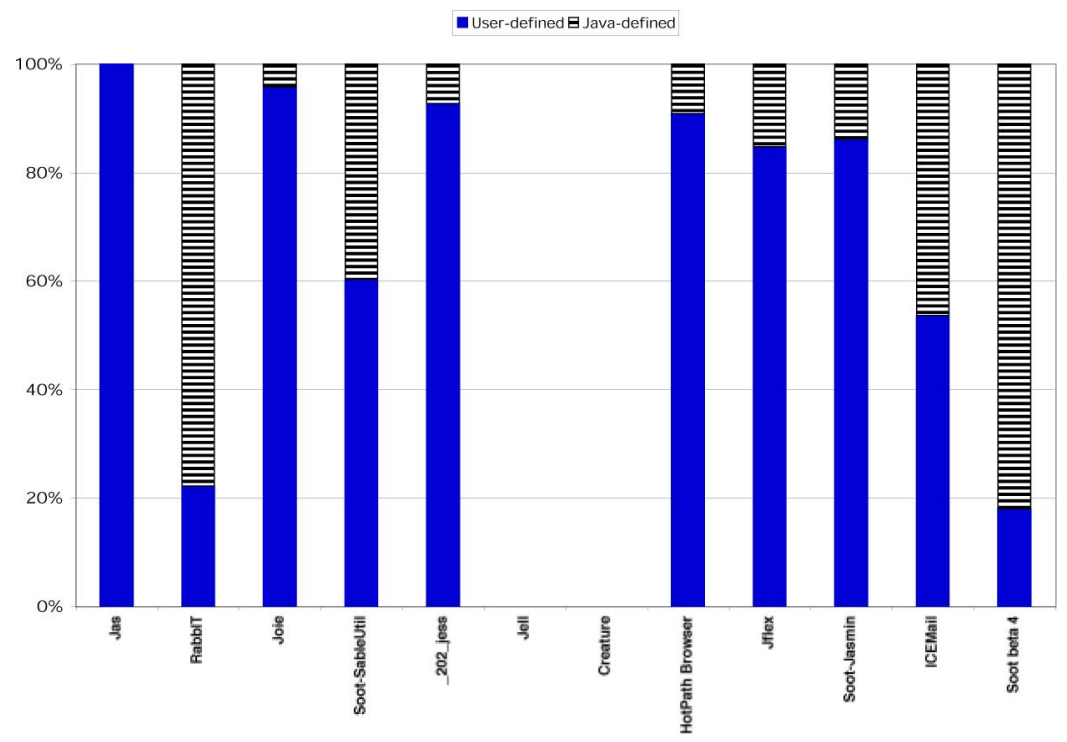

Fig. 8. User-defined versus Java-defined exceptions

timize the execution of control exceptions and should try to limit the effects of all exceptions on the safety and benefits of other transformations.

\section{Possible Optimizations}

Knowing statically the binding between a thrown exception and the catch clause that will process the exception has two advantages that can be exploited by an optimizing compiler:

- No runtime check is necessary to determine if the next current method will handle the exception.

- The code contained in the corresponding catch clause can be moved to the site where the exception is thrown, allowing better code locality. In addition, the runtime stack can be updated with a single multi-frame pop operation. This optimization assumes that no statement such as a finally occurs in any intermediate method invocation between the throw and catch methods. This safety property can be easily verified by a compile-time analysis.

\section{Related Work}

There has been much recent interest in the problems presented to compilation and analysis of programs by Java exceptions. Of course, any Java compiler must handle exceptions; for space reasons all Java compilers under development cannot be presented here. Therefore, this discussion focuses on research which reports exception usage. 
Sinha and Harrold[10] present new control-flow representations to model exception flow for data-flow and control-dependence analyses. They performed a quick static study of a seven Java programs and found that that on average $23 \%$ of their methods contained a try or a throw. Their dataset included: jacorb, javacup, jdk, jlex, swing, tdb, and toba. They use this data to substantiate the hypothesis that analysis algorithms will have to take account of exceptions in Java because of their prevalence.

Chatterjee, Ryder and Landi[5] presented a new flow-sensitive analysis, called relevant context inference $(R C I)$ for a subset of Java that can model exception control flow. This analysis stresses optimized memory usage by only requiring the code for a method to be in memory three times during the analysis. Exception control flow is modeled as another context preserved by the algorithm (with alias and type contexts). Chatterjee's thesis[4] shows how to use RCI to calculate defuse associations in the presence of exceptions.

Krall and Probst[13] performed a small static study of five Java programs (i.e., JavaLex, javac, espresso, Toba, java_cup) used as data for their $C A$ $C A O$ compiler. They measured number of try blocks versus number of method calls in these programs and found that the latter was two orders of magnitude larger than the former. They also reported on the number of null pointer checks in these programs which was more comparable to the number of method invocations.

Choi et.al.[6] present a new intermediate representation, a factored control flow graph, used in the IBM Jalapeno dynamic Java compiler that accommodates exits caused by possible implicit exceptions such as NullPointerException. They report static counts of the number of basic blocks with exception handler edges for implicit exceptions, both for the traditional basic block and their new design.

The Marmot optimizing Java compiler from Microsoft[7] also optimizes implicit exceptions. Their transformations make it possible to catch NullPointerExceptions using hardware.

Robaillard and Murphy[14] report on Jex, a static tool for identifying userdefined exception control flow in Java programs. Given a Java source file and the user-called packages (denoted by the user), Jex derives a representation of exception flow through the program. Exceptions generated by the Java API were also counted. For each Java method, Jex produces a summary of those exceptions possibly thrown by its execution. The empirical study reported focused on the use of subsumption in catch clauses. About $44 \%$ of the exceptions within try blocks were not caught with the most precise exception type available. The goal was to give this information to a programmer to increase their understanding of possible exception flow in their program.

Brookshier[3] measured and reported on the cost of exception processing for control exceptions when the handler is not close to the method throwing the exception on the runtime stack. He describes the cost of a throw as due to creation of an exception object, an invocation of the handler, a search of the stack for the current method, a search for a try block range, and then the search for a matching catch. He notes that cost of a throw is related to the depth of nested 
frames, so that speed can be improved if the catch clause is in the same method or relatively close on the runtime stack. Measured on a $300 \mathrm{MHz}$ Pentium II with Symantec's 1.1 JIT, there was a slowdown of 80 milliseconds per exception in a program written so that the handler is several stack frames from the throw.

\section{Conclusions and Future Work}

The studies reported here with the Prolangs JESP tool are an attempt to discern the generality with which explicit exception constructs (i.e., try, catch, finally, throw) occur in Java codes. The most important results were that a substantial percentage of methods (on average 16\%) in a Java program contain exception constructs, so exception usage is ubiquitous, but exception constructs are fairly sparse (the number of trys match number of catches). Finallys are rare and thrown exceptions are usually not caught within the same method. Userdefined exception hierarchies are shallow. Another interesting finding was the observed dramatic categorization of programs which display many more throws than catches and vice versa.

As with all initial studies, this one raises more questions than it answers. Future work includes measurement of the:

- dynamic behavior of user-thrown exception,

- use of control and abortive exceptions,

- number of user- and Java-defined exceptions caught, and

- number of times a catch clause catches an exception thrown from within the try block.

Acknowledgments. We sincerely thank Seth Cohen from Compaq Corporation whose help with $\mathbf{J}$ Trek was invaluable.

\section{References}

1. K. Arnold and J. Gosling. The Java Programming Language, Second Edition. Addison-Wesley, 1997. 68

2. M. Arnold, M. Hsiao, U. Kremer, and B.G. Ryder. Instruction scheduling in the presence of java's runtime exceptions. In Proceedings of Workshop on Languages and Compilers for Parallel Computation (LCPC'99), August 1999. 68

3. D. Brookshier. Exception handling: Simpler, faster, safer. Java Report, 3(2), February 1998. 79

4. R. Chatterjee. Modular Data-flow Analysis of Statically Typed Object-oriented Programming Languages. PhD thesis, Department of Computer Science, Rutgers University, October 1999. 68, 79

5. Ramkrishna Chatterjee, Barbara G. Ryder, and William. A Landi. Relevant context inference. In Conference Record of the Twenty-sixth Annual ACM SIGACT/SIGPLAN Symposium on Principles of Programming Languages, January $1999 . \quad 68,79$ 
6. J.-D. Choi, D. Grove, M. Hind, and V. Sarkar. Efficient and precise modeling of exceptions for the analysis of java programs. In Proceedings of the ACM SIGPLAN/SIGSOFT Workshop on Program Analysis for Software Tools and Engineering, pages 1-11, September 1999. 68, 79

7. R. Fitzgerald, T.B. Knoblock, E. Rif, B. Steensgaard, and D. Tarditi. Marmot: An optimizing compilr for java. Technical Report Technical Report MSR-TR-99-33, Microsoft Research, June 1999. 68, 79

8. Andrew Gelsey, Don Smith, Mark Schwabacher, Khaled Rasheed, and Keith Miyake. A search space toolkit. Decision Support Systems - special issue on Unification of Artificial Intelligence with Optimization, 18:341-356, 1996. 68

9. J. Gosling, B. Joy, and G. Steele. The Java Language Specification. AddisonWesley, 1996. 68

10. M.J. Harrold and S. Sinha. Analysis of programs with exception-handling constructs. In Proceedings of the International Conference on Software Maintenance, November 1998. 68, 79

11. J. Keane. Knowledge-based Management of Legacy Codes for Automated Design. PhD thesis, Rutgers University, October 1996. 68

12. J. Keane and T. Ellman. Knowledge-based re-engineering of legacy programs for robustness in automated designs. In Proceedings of the Eleventh Knowledge-Based Software Engineering Conference, 1996. 68

13. A. Krall and M. Probst. Monitors and exceptions: How to implement java efficiently. In Proceedings of 1998 ACM Java Workshop, 1998. 68, 79

14. M. Robillard and G. Murphy. Analyzing exception flow in java programs. In Proceedings of the rth Annual ACM SIGSOFT Symposium on the Foundations of Software Engineering, September 1999. 68, 79

15. Raja Vallee-Rai, Laurie Hendren, Vijay Sundaresan, Patrick Lamand Etienne Gagnon, and Phong Co. Soot - a java optimization framework. In CASCON99, Toronto, Ontario, September 1999. 69 